\title{
Anuric Renal Failure after Same-Session Bilateral Atraumatic Flexible Ureteroscopy
}

\author{
Gaurav Bandi, Fabio C. Vicentini, Jeffrey A. Triest \\ Department of Urology, Wayne State University, Detroit, Michigan, USA
}

\begin{abstract}
We report the first known case of anuric renal failure after same-session bilateral atraumatic flexible ureteroscopy for renal calculi. Although, there is no consensus about stenting patients who undergo same-session bilateral ureteroscopy due to the lack of prospective randomized studies; strong consideration should be given to stenting the ureter at least one side to avoid this complication.
\end{abstract}

Key words: ureteroscopy; complications; renal failure; anuria

Int Braz J Urol. 2007; 33: 193-4

\section{INTRODUCTION}

The use of flexible ureteroscope for the treatment of renal stones has success rates from 50$92 \%$ (1). Same-session bilateral ureteroscopy has been shown to be safe and effective; however, it is associated with a higher complication rate if compared to staged bilateral ureteroscopy (2). We report the first known case of anuric renal failure after samesession bilateral flexible ureteroscopy for renal calculi.

\section{CASE REPORT}

A 53-year-old African-American female with a known history of nephrolithiasis was found to have symptomatic, bilateral, small renal calculi on CT scan. Under general anesthesia, bilateral flexible ureteroscopy was performed by passing the $6.9 \mathrm{~F}$ ureteroscope over hydrophilic guide wire in an atraumatic fashion without the need for ureteral dilatation. On the right side, she was found to have a $5 \mathrm{~mm}$ lower pole and an $8 \mathrm{~mm}$ mid pole calculus behind a stenotic infundibulum. Infundibulotomy was carried out using a $200 \mathrm{~nm}$ holmium laser fiber followed by stone fragmentation. Diagnostic left ureteroscopy revealed calcified renal papillae. The procedure was atraumatic on both sides and ureteral integrity was confirmed while taking the ureteroscope out. The procedure took 60 minutes on the right and 10 minutes on the left side.

Over the next 24 hours, the patient developed anuric renal failure (elevation of serum creatinine from 0.9 to $3.5 \mathrm{mg} / \mathrm{dL}$ ) and symptomatic pulmonary edema due to fluid overload. Emergent bilateral ureteral stents were placed. Over the next 48 hours, she had 
hematuria and post-obstructive diuresis with normalization of her creatinine level and resolution of pulmonary edema. She was eventually discharged home 6 days after her surgery.

\section{COMMENTS}

Ureteral obstruction is a known complication following ureteroscopy and may be secondary to residual stones, clots, ureteral stricture, ureteral spasm or mucosal edema (3). Postoperative pain and renal colic has been reported in 4-9\% of patients undergoing ureteroscopic procedures (3). Obstruction is usually self-limited and can be managed by conservative measures.

Routine stenting after unilateral atraumatic ureteroscopic lithotripsy of stones has not shown to be beneficial in many randomized controlled trials (4). However, these studies are limited due to their strict inclusion criteria. Factors associated with increased postoperative morbidity include renal pelvis location, lithotripsy, bilateral procedure and increased operative time (4).

Same-session bilateral ureteroscopy has been proposed to reduce overall operative time, anesthetic requirements and time to convalescence (2). It has been shown to be associated with increased morbidity compared to staged bilateral ureteroscopy (29\% vs. $14 \%$ ); however, there was no difference in cumulative morbidity and stone free rates at 1 month between the two approaches (2). Although, placement of ureteral stent has been recommended by some authors $(2,4)$; there is no consensus about stenting patients who undergo same-session bilateral ureteroscopy.

Our case is unique as the procedure was short, completely uneventful and atraumatic on both sides. The exact etiology of ureteral obstruction is unknown but it is likely related to ureteral edema and spasm from instrumentation.

\section{CONCLUSION}

Bilateral atraumatic ureteroscopy can be associated with anuric renal failure in rare cases.
Strong consideration should be given to stenting the ureter at least one side to avoid this complication.

\section{CONFLICT OF INTEREST}

None declared.

\section{REFERENCES}

1. Busby JE, Low RK: Ureteroscopic treatment of renal calculi. Urol Clin North Am. 2004; 31: 89-98.

2. Hollenbeck BK, Schuster TG, Faerber GJ, Wolf JS Jr: Safety and efficacy of same-session bilateral ureteroscopy. J Endourol. 2003; 17: 881-5.

3. Johnson DB, Pearle MS: Complications of ureteroscopy. Urol Clin North Am. 2004; 31: 157-71.

4. Hollenbeck BK, Schuster TG, Seifman BD, Faerber GJ, Wolf JS Jr: Identifying patients who are suitable for stentless ureteroscopy following treatment of urolithiasis. J Urol. 2003; 170: 103-6.

Accepted after revision:

September 15, 2006 